Article

\title{
Horadam Sequences and Tridiagonal Determinants
}

\section{Kwang-Wu Chen}

Department of Mathematics, University of Taipei, No. 1, Ai-Guo West Road, Taipei 10048, Taiwan; kwchen@uTaipei.edu.tw

Received: 10 November 2020; Accepted: 26 November 2020; Published: 28 November 2020

Abstract: We consider a family of particular tridiagonal matrix determinants which can represent the general second-order linear recurrence sequences. These determinants can be changed to symmetric or skew-symmetric tridiagonal determinants. To evaluate the complex factorizations of any Horadam sequence, we evaluate the eigenvalues of some special tridiagonal matrices and their corresponding eigenvectors. We also use these determinant representations to obtain some formulas in these sequences.

Keywords: Horadam sequences; second-order linear recurrence sequences; tridiagonal determinants; eigenvalues

MSC: 11B39; 15A15; 15A18; 47B36

\section{Introduction}

The general second-order linear homogeneous recurrence relation is

$$
w_{n}=p w_{n-1}-q w_{n-2}, \quad w_{0}=a, w_{1}=b .
$$

Sometimes we call this sequence as a Horadam sequence after the work of Horadam [1] and is denoted by $\mathbb{W}(a, b ; p, q)=\left(w_{n}(a, b ; p, q)\right)$.We usually omit the $(a, b ; p, q)$ if it does not cause ambiguity. Let $\mathbb{U}(p, q)=\left(U_{n}(p, q)\right)$ and $\mathbb{V}(p, q)=\left(V_{n}(p, q)\right)$ denote the Lucas sequence of the first kind and the second kind, respectively, which are defined by

$$
U_{n}(p, q)=w_{n}(0,1 ; p, q) \text { and } V_{n}(p, q)=w_{n}(2, p ; p, q)
$$

Fibonacci numbers $F_{n}=U_{n}(1,-1)$, Lucas numbers $L_{n}=V_{n}(1,-1)$, Pell numbers $P_{n}=U_{n}(2,-1)$, Pell-Lucas numbers $Q_{n}=V_{n}(2,-1)$, Jacobsthal numbers $J_{n}=U_{n}(1,-2)$, and Jacobsthal-Lucas numbers $j_{n}=V_{n}(1,-2)$ are some most famous particular cases of Lucas sequences.

There are many known connections between tridiagonal matrix determinants and Fibonacci and Lucas numbers. Some examples follow. Strang and Borre [2] and Strang [3]:

$$
F_{n+1}=\operatorname{det}\left[\begin{array}{ccccc}
1 & -1 & & & \\
1 & 1 & & & \\
& & \ddots & & \\
& & & 1 & -1 \\
& & & 1 & 1
\end{array}\right]_{n \times n}, F_{2 n+1}=\operatorname{det}\left[\begin{array}{ccccc}
2 & -1 & & & \\
-1 & 3 & & & \\
& & \ddots & & \\
& & 3 & -1 \\
& & -1 & 3
\end{array}\right]_{n \times n}
$$


Cahill et al. [4]:

$$
F_{n+1}=\operatorname{det}\left[\begin{array}{ccccc}
1 & i & & & \\
i & 1 & & & \\
& & \ddots & & \\
& & & 1 & i \\
& & & i & 1
\end{array}\right]_{n \times n}, L_{n}=\operatorname{det}\left[\begin{array}{cccccc}
1 & i & & & & \\
i & 2 & i & & & \\
& i & 1 & & & \\
& & & \ddots & & \\
& & & & 1 & i \\
& & & & i & 1
\end{array}\right]_{n \times n}
$$

Cahill and Narayan [5]:

$$
F_{3 n+3}=\operatorname{det}\left[\begin{array}{cccccc}
8 & \sqrt{6} & & & & \\
\sqrt{6} & 5 & i & & & \\
& i & 4 & & & \\
& & & \ddots & & \\
& & & & 4 & i \\
& & & & i & 4
\end{array}\right]_{n \times n}
$$

In this paper, we systematically study the relationship between Horadam sequences and tridiagonal determinants. In fact, we investigate the following $n \times n$ tridiagonal matrices, which we denote $M_{n}\left(\begin{array}{l}a, b, c \\ d, e, f\end{array}\right)$. The elements of $M_{n}\left(\begin{array}{l}a, b, c \\ d, e, f\end{array}\right)$ are given by $m_{1,1}=a, m_{1,2}=b, m_{2,1}=c, m_{\ell, \ell}=d$, where $2 \leq \ell \leq n$, and $m_{k, k+1}=e, m_{k+1, k}=f$, where $2 \leq k \leq n-1$. That is,

$$
M_{n}\left(\begin{array}{l}
a, b, c, c \\
d, e, f
\end{array}\right)=\left[\begin{array}{cccccc}
a & b & & & & \\
c & d & e & & & \\
& f & d & & & \\
& & & \ddots & & \\
& & & & d & e \\
& & & & f & d
\end{array}\right]_{n \times n}
$$

We also define the $n \times n$ tridiagonal Toeplitz matrices by

$$
\mathbb{M}_{n}(a, b, c)=M_{n}\left(\begin{array}{c}
a, b, c \\
a, b, c
\end{array}\right) .
$$

Their corresponding determinants are denoted as

$$
D_{n}\left(\begin{array}{l}
a, b, c \\
d, e, f
\end{array}\right)=\operatorname{det} M_{n}\left(\begin{array}{l}
a, b, c, c \\
d, e, f
\end{array}\right) \quad \text { and } \quad \mathbb{D}_{n}(a, b, c)=\operatorname{det} \mathbb{M}_{n}(a, b, c) .
$$

It is clear that, if we write Equation (1) as our notations, they are

$$
F_{n+1}=\mathbb{D}_{n}(1,-1,1) \quad \text { and } \quad F_{2 n+1}=D_{n}\left(\begin{array}{c}
2,-1,-1 \\
3,-1,-1
\end{array}\right) .
$$

In fact, these results can be covered by our theorems. Horadam sequences have the determinantal representations as follows.

$$
w_{n}(a, b ; p, q)=D_{n+1}\left(\begin{array}{c}
a, 1, a p-b \\
p, 1,
\end{array}\right)=D_{n}\left(\begin{array}{l}
b, a, q \\
p, 1, q
\end{array}\right),
$$

for a positive integer $n$ and any complex numbers $a, b, p, q$ (see Theorem 1). 
We use these determinantal representations of $w_{n}(a, b ; p, q)$ and the basic properties of determinants to get some identities among Horadam sequences. For example (see [1] [Equation (3.14)]),

$$
w_{m}(a, b ; p, q) U_{n+1}(p, q)-q w_{m-1}(a, b ; p, q) U_{n}(p, q)=w_{m+n}(a, b ; p, q)
$$

Here, we give a new formula.

$$
w_{-n}(a, b ; p, q)=q^{-n_{w}} w_{n}(a, a p-b ; p, q)
$$

Note that there is another formula obtained by the author and Pan [6] [Equation (10)]. Moreover, since the determinant of a matrix can be found by the product of all its eigenvalues. Cahill, D'Errico, and Spence [7] used this fact to get the complex factorization of Fibonacci numbers $F_{n}$ :

$$
F_{n}=\prod_{k=1}^{n-1}\left(1-2 i \cos \frac{k \pi}{n}\right)
$$

for $n \geq 2$. Here, we extend this result to any Horadam sequences. For example, we have

$$
U_{k n+k}=U_{k} \prod_{j=1}^{n}\left(V_{k}+2 \sqrt{q^{k}} \cos \frac{j \pi}{n+1}\right)
$$

Setting the parameters $p=-q=1$ and $k=2 m$, we have [5] [Equation (3)]

$$
F_{2 m(n+1)}=F_{2 m} \prod_{j=1}^{n}\left(L_{2 m}+2 \cos \frac{j \pi}{n+1}\right)
$$

where $m, n$ are integers and $n \geq 1$. In fact, Equation (8) gives more formulas, including

$$
P_{n+1}=\prod_{j=1}^{n}\left(2+2 i \cos \frac{j \pi}{n+1}\right), \quad \text { and } \quad P_{2 n+2}=2 \prod_{j=1}^{n}\left(6+2 \cos \frac{j \pi}{n+1}\right) \text {. }
$$

This paper is organized as follows. In Section 2, we introduce some basic properties of the determinant $D_{n}\left(\begin{array}{l}a, b, c \\ d, e, f\end{array}\right)$. In Section 3 , we transform $w_{n}(a, b ; p, q)$ to its corresponding determinant $D_{n+1}\left(\begin{array}{c}a, 1, a p-b \\ p, 1,\end{array}\right.$, . Then, we use some properties of determinants to derive some well-known identities among Horadam sequences. In Section 4 , we evaluate the eigenvalues of the matrix $M_{n}\left(\begin{array}{c}m, 1, q \\ p, 1, q\end{array}\right)$ and their corresponding eigenvectors in order to give the complex factorizations of any Horadam sequences. In Section 5, we give explicitly the complex factorizations of the Lucas sequences of the first kind and the second kind. In Section 6, we substitute some particular integers into the formulas in Section 5 to give some expressions among some famous sequences: Fibonacci numbers $F_{n}$, Lucas numbers $L_{n}$, Pell numbers $P_{n}$, and Pell-Lucas numbers $Q_{n}$.

\section{Preliminaries}

We list some basic properties of $D_{n}\left(\begin{array}{l}a, b, c \\ d, e, f\end{array}\right)$ :

$$
\begin{gathered}
D_{n}\left(\begin{array}{c}
a, 0, c \\
d, e, f
\end{array}\right)=D_{n}\left(\begin{array}{c}
a, b, 0 \\
d, e, f
\end{array}\right)=a \mathbb{D}_{n-1}(d, e, f), \\
\alpha D_{n}\left(\begin{array}{c}
a, b, c \\
d, e, f
\end{array}\right)=D_{n}\left(\begin{array}{c}
a \alpha, b \alpha, c \\
d, e, f
\end{array}\right)=D_{n}\left(\begin{array}{c}
a \alpha, b, c \alpha \\
d, e, f
\end{array}\right) .
\end{gathered}
$$

Since $M_{n}\left(\begin{array}{l}a, 0, c \\ d, e, f\end{array}\right)$ and $M_{n}\left(\begin{array}{l}a, b, 0 \\ d, e, f\end{array}\right)$ have only one nonzero element in the first row and in the first column, respectively, if we expand them along the row or the column, Equation (9) will be obtained. Equation (10) is resulted from multiplying the first row or the first column by $\alpha$. 
From the basic operations of determinants, we also have

$$
\begin{aligned}
\alpha^{n} D_{n}\left(\begin{array}{c}
a, b, c \\
d, e, f
\end{array}\right) & =D_{n}\left(\begin{array}{c}
\alpha a, \alpha b, \alpha c \\
\alpha d, \alpha e, \alpha f
\end{array}\right), \\
D_{n}\left(\begin{array}{c}
a, b, c, c \\
d, e, f
\end{array}\right)+D_{n}\left(\begin{array}{c}
a^{\prime}, b^{\prime}, c \\
d, e, f
\end{array}\right) & =D_{n}\left(\begin{array}{c}
a+a^{\prime}, b+b^{\prime}, c \\
d, \\
e, f
\end{array}\right), \\
D_{n}\left(\begin{array}{c}
a, b, c, c \\
d, e, f
\end{array}\right)+D_{n}\left(\begin{array}{c}
a^{\prime}, b, c^{\prime} \\
d, e, f
\end{array}\right) & =D_{n}\left(\begin{array}{c}
a+a^{\prime}, b, c+c^{\prime} \\
d, e, f
\end{array}\right) .
\end{aligned}
$$

We evaluate $D_{n}\left(\begin{array}{l}a, b, c \\ d, e, f\end{array}\right)$ by using the Laplace expansion along the first row; we have

$$
D_{n}\left(\begin{array}{l}
a, b, c \\
d, e, f
\end{array}\right)=a \mathbb{D}_{n-1}(d, e, f)-b D_{n-1}\left(\begin{array}{l}
c, e, 0 \\
d, e, f
\end{array}\right) .
$$

Applying Equation (9) to the above identity, we have

$$
D_{n}\left(\begin{array}{l}
a, b, c, c \\
d, e, f
\end{array}\right)=a \mathbb{D}_{n-1}(d, e, f)-b c \mathbb{D}_{n-2}(d, e, f) .
$$

On the other hand, if we evaluate $D_{n}\left(\begin{array}{l}a, b, c, c \\ d, e, f\end{array}\right)$ by using the Laplace expansion from the bottom, we have the recurrence relation:

$$
D_{n}\left(\begin{array}{l}
a, b, c \\
d, e, f
\end{array}\right)=d D_{n-1}\left(\begin{array}{l}
a, b, c \\
d, e, f
\end{array}\right)-\text { ef } D_{n-2}\left(\begin{array}{l}
a, b, c \\
d, e, f
\end{array}\right) .
$$

All the above determinant properties are basic (see [2,3]); we only use our notations to represent them, which is convenient for the subsequent use of this paper.

Lemma 1. If $b c=b^{\prime} c^{\prime}$ and $e f=e^{\prime} f^{\prime}$, then

$$
D_{n}\left(\begin{array}{l}
a, b, c, c \\
d, e, f
\end{array}\right)=D_{n}\left(\begin{array}{l}
a, b^{\prime}, c^{\prime} \\
d, e^{\prime}, f^{\prime}
\end{array}\right)
$$

Proof. Using Equation (14) and $b c=b^{\prime} c^{\prime}$, we have

$$
\begin{aligned}
D_{n}\left(\begin{array}{c}
a, b, c \\
d, e, f
\end{array}\right) & =a \mathbb{D}_{n-1}(d, e, f)-b c \mathbb{D}_{n-2}(d, e, f) \\
& =a \mathbb{D}_{n-1}(d, e, f)-b^{\prime} c^{\prime} \mathbb{D}_{n-2}(d, e, f) \\
& =D_{n}\left(\begin{array}{c}
a, b b^{\prime}, c^{\prime} \\
d, e, f
\end{array}\right) .
\end{aligned}
$$

Then, we use induction on $n$ to prove that $D_{n}\left(\begin{array}{c}a, b^{\prime}, c^{\prime} \\ d, e, f\end{array}\right)=D_{n}\left(\begin{array}{c}a, b^{\prime}, c^{\prime} \\ d, e^{\prime}, f^{\prime}\end{array}\right)$. For the trivial case $D_{1}\left(\begin{array}{c}a, b^{\prime}, c^{\prime} \\ d, e, f\end{array}\right)=$ $a=D_{1}\left(\begin{array}{c}a, b^{\prime}, c^{\prime} \\ d, e^{\prime}, f^{\prime}\end{array}\right)$ and $D_{2}\left(\begin{array}{c}a, b^{\prime}, c^{\prime} \\ d, e, f\end{array}\right)=a d-b^{\prime} c^{\prime}=D_{2}\left(\begin{array}{c}a, b^{\prime}, c^{\prime} \\ d, e^{\prime}, f^{\prime}\end{array}\right)$. For $n \geq 3$, we assume that this identity is true for $n-1$ and $n-2$. Applying the recurrence relation Equation (15), we have

$$
\begin{aligned}
D_{n}\left(\begin{array}{c}
a, b^{\prime}, c^{\prime} \\
d, e, f
\end{array}\right) & =d D_{n-1}\left(\begin{array}{c}
a, b^{\prime}, c^{\prime} \\
d, e, f
\end{array}\right)-e f D_{n-2}\left(\begin{array}{c}
a, b^{\prime}, c^{\prime} \\
d, e, f
\end{array}\right) \\
& =d D_{n-1}\left(\begin{array}{c}
a, b^{\prime}, c^{\prime} \\
d, e^{\prime}, f^{\prime}
\end{array}\right)-e^{\prime} f^{\prime} D_{n-2}\left(\begin{array}{c}
a, b^{\prime}, c^{\prime}, c^{\prime}, e^{\prime}, f^{\prime} \\
y^{\prime}
\end{array}\right)=D_{n}\left(\begin{array}{c}
a, b^{\prime}, c^{\prime} \\
d, e^{\prime}, f^{\prime}
\end{array}\right) .
\end{aligned}
$$

Using this lemma, we can transform $D_{n}\left(\begin{array}{l}a, b, c, c \\ d, e, f\end{array}\right)$ to a symmetric tridiagonal determinant $D_{n}\left(\begin{array}{l}a, \sqrt{b c}, \sqrt{b c} \\ d, \sqrt{e f}, \sqrt{e f}\end{array}\right)$ or a skew-symmetric tridiagonal determinant $D_{n}\left(\begin{array}{l}a, i \sqrt{b c},-i \sqrt{b c} \\ d, i \sqrt{e f},-i \sqrt{e f}\end{array}\right)$.

We reduce the order $n$ of $D_{n}\left(\begin{array}{l}a, b, c, c \\ d, e, f\end{array}\right)$ by the following identity.

Lemma 2. For any positive integer $n$,

$$
D_{n+1}\left(\begin{array}{c}
a, b, c \\
d, e, f
\end{array}\right)=D_{n}\left(\begin{array}{cc}
a d-b c, a e, f \\
d, & e, f
\end{array}\right) .
$$


Proof. If $a \neq 0$, then we multiply $a$ to the second row.

$$
D_{n}\left(\begin{array}{l}
a, b, c, c \\
d, e, f
\end{array}\right)=\frac{1}{a} \operatorname{det}\left[\begin{array}{cccccc}
a & b & & & & \\
a c & a d & a e & & & \\
& f & d & & & \\
& & & \ddots & & \\
& & & & d & e \\
& & & & f & d
\end{array}\right] .
$$

Multiplying $(-c)$ to the first row and adding it to the second row:

$$
D_{n}\left(\begin{array}{l}
a, b, c \\
d, e, f
\end{array}\right)=\frac{1}{a} \operatorname{det}\left[\begin{array}{cccccc}
a & b & & & & \\
& a d-b c & a e & & & \\
& f & d & & & \\
& & & \ddots & & \\
& & & & d & e \\
& & & & f & d
\end{array}\right] .
$$

Expanding the determinant along the first column, we have the desired formula. If $a=0$, we use Equation (14) and get

$$
D_{n+1}\left(\begin{array}{l}
0, b, c \\
d, e, f
\end{array}\right)=-b c \mathbb{D}_{n-1}(d, e, f) .
$$

On the other hand, we use Equation (9) and get

$$
D_{n}\left(\begin{array}{cc}
a d-b c, a e, f \\
d, & e, f
\end{array}\right)=D_{n}\left(\begin{array}{c}
-b c, 0, f \\
d, e, f
\end{array}\right)=-b c \mathbb{D}_{n-1}(d, e, f) .
$$

Therefore, we get Equation (17).

The following is a quick result of the above theorem.

Corollary 1. For any positive integer $n$,

$$
\text { ef } D_{n}\left(\begin{array}{l}
a, b, c, c \\
d, e, f
\end{array}\right)=D_{n+1}\left(\begin{array}{cc}
b c, 1, b c d-a e f \\
d, e, & f
\end{array}\right) .
$$

\section{Some Properties of Horadam Sequences}

Theorem 1. For $\alpha \neq 0 \neq \beta$, we have

$$
w_{n}(a, b ; p, q)=D_{n+1}\left(\begin{array}{cc}
a, \beta, & (a p-b) / \beta \\
p, \alpha, & q / \alpha
\end{array}\right) .
$$

In particular, if we set $\alpha=\beta=1$ in the above identity, then

$$
w_{n}(a, b ; p, q)=D_{n+1}\left(\begin{array}{c}
a, 1, a p-b \\
p, 1,
\end{array}\right)=D_{n}\left(\begin{array}{l}
b, a, q \\
p, 1, q
\end{array}\right) .
$$

Proof. First, we observe that the values

$$
D_{1}\left(\begin{array}{c}
a, \beta_{1}(a p-b) / \beta \\
p, \alpha, \underset{q / \alpha}{\alpha}
\end{array}\right)=a=w_{0}, \quad D_{2}\left(\begin{array}{c}
a, \beta_{1}(a p-b) / \beta \\
p, \alpha_{\alpha}, \underset{q / \alpha}{q}
\end{array}\right)=b=w_{1} .
$$


Therefore, these two sequences $\left(D_{n+1}\right)$ and $\left(w_{n}\right)$ have the same initial values. Using Equation (15), we have the recurrence relation of the sequence $\left(D_{n+1}\right)$ :

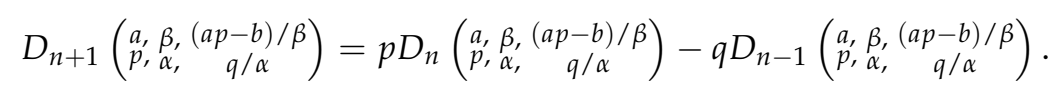

It appears that the sequence $\left(D_{n+1}\right)$ has the same recurrence relation with $\left(w_{n}\right)$. Thus, for all integer $n \geq 0$,

$$
D_{n+1}\left(\begin{array}{c}
a, \beta, \\
p, \alpha, \underset{q / \alpha}{(a p-b) / \beta}
\end{array}\right)=w_{n} .
$$

The particular identities are substituted by $\alpha=\beta=1$ and Equation (17) is applied to the above determinant.

Using Equation (20), we can get the determinantal representations of the sequence $\left(U_{n}(p, q)\right)$ and $\left(V_{n}(p, q)\right)$.

$$
\begin{aligned}
& U_{n}(p, q)=w_{n}(0,1 ; p, q)=D_{n}\left(\begin{array}{l}
1,0, q \\
p, 1, q
\end{array}\right)=\mathbb{D}_{n-1}(p, 1, q), \\
& V_{n}(p, q)=w_{n}(2, p ; p, q)=D_{n}\left(\begin{array}{c}
p, 2, q \\
p, 1, q
\end{array}\right) .
\end{aligned}
$$

Applying Equations (14) and (21), we have the well-known identity [1] [Equation (2.14)]

$$
\begin{aligned}
w_{n}(a, b ; p, q) & =D_{n}\left(\begin{array}{l}
b, a, q \\
p, 1, q
\end{array}\right) \\
& =b \mathbb{D}_{n-1}(p, 1, q)-a q \mathbb{D}_{n-2}(p, 1, q) \\
& =b U_{n}(p, q)-a q U_{n-1}(p, q) .
\end{aligned}
$$

Proposition 1. For any nonnegative integer $n$, we have

$$
\begin{aligned}
& w_{n}(a, b ;-p, q)=(-1)^{n} w_{n}(a,-b ; p, q), \\
& w_{-n}(a, b ; p, q)=q^{-n} w_{n}(a, a p-b ; p, q) .
\end{aligned}
$$

Proof. Using Equation (20) and Lemma 1, we get

$$
\begin{aligned}
w_{n}(a, b ;-p, q) & =D_{n}\left(\begin{array}{c}
b, a, q \\
-p, q, q
\end{array}\right) \quad=(-1)^{n} D_{n}\left(\begin{array}{c}
-b,-a,-q \\
p,-1,-q
\end{array}\right) \\
& =(-1)^{n} D_{n}\left(\begin{array}{c}
-b, a, q \\
p, 1, q
\end{array}\right)=(-1)^{n} w_{n}(a,-b ; p, q) .
\end{aligned}
$$

Now, we substitute $-n$ into the recurrence relation of the sequence $\left(w_{n}(a, b ; p, q)\right)$.

$$
\begin{aligned}
w_{-n} & =p w_{-n-1}-q w_{-n-2} \\
q w_{-(n+2)} & =p w_{-(n+1)}-w_{-n} .
\end{aligned}
$$

Therefore, the sequence $\left(w_{-n}\right)$ satisfies the recurrence relation:

$$
w_{-n}=\frac{p}{q} w_{-(n-1)}-\frac{1}{q} w_{-(n-2)}
$$

The initial values $w_{0}=a$ and

$$
w_{-1}=p / q \cdot w_{0}-1 / q \cdot w_{1}=p a / q-b / q=(p a-b) / q .
$$

Thus, we have

$$
w_{-n}(a, b ; p, q)=w_{n}(a,(p a-b) / q ; p / q, 1 / q) .
$$


Again, if we use Equation (20) and Lemma 1, then we have

$$
\begin{aligned}
& w_{-n}(a, b ; p, q)=w_{n}(a,(a p-b) / q ; p / q, 1 / q)=D_{n}\left(\begin{array}{cc}
(a p-b) / q, a, 1 / q \\
p / q, & 1,1 / q
\end{array}\right) \\
& =\frac{1}{q^{n}} D_{n}\left(\begin{array}{c}
a p-b, a q, 1 \\
p, q, 1
\end{array}\right) \quad=\frac{1}{q^{n}} D_{n}\left(\begin{array}{c}
a p-b, a, q \\
p, \\
1, q
\end{array}\right) \\
& =q^{-n} w_{n}(a, a p-b ; p, q) \text {. }
\end{aligned}
$$

This completes our proof.

Note that there is another formula obtained by the author and Pan [6] [see Equation (10)]:

$$
\begin{aligned}
q^{-(n+1)} w_{n+1}(b, q a ; p, q)=q^{-(n+1)} D_{n+1}\left(\begin{array}{c}
q a, b, q \\
p, 1, q
\end{array}\right) & =q^{-(n+1)} D_{n}\left(\begin{array}{c}
a p q-b q, a q, q \\
p, \\
1, q
\end{array}\right) \\
=q^{-n} D_{n}\left(\begin{array}{c}
a p-b, a, q \\
p, 1, q
\end{array}\right) & =q^{-n} w_{n}(a, a p-b ; p, q) \\
& =w_{-n}(a, b ; p, q) .
\end{aligned}
$$

We list the formulas of the sequences $\left(U_{-n}(p, q)\right)$ and $\left(V_{-n}(p, q)\right.$ (see [1] [Equation (2.17), (2.19)]):

$$
\begin{aligned}
& U_{-n}(p, q)=q^{-n} w_{n}(0,-1 ; p, q)=-q^{-n} U_{n}(p, q), \\
& V_{-n}(p, q)=q^{-n} w_{n}(2, p ; p, q)=q^{-n} V_{n}(p, q) .
\end{aligned}
$$

Proposition 2. Given a sequence $\left(w_{n}(a, b ; p, q)\right)$, where $a, b, p, q$ are arbitrary complex numbers, and $n$ is an integer, for any integer $m, k$ with $m \geq 0$, we have

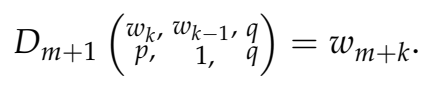

Proof. Using Equation (17), we have

$$
D_{m+1}\left(\begin{array}{ccc}
w_{k}, w_{k-1}, q \\
p, & 1, & q
\end{array}\right)=D_{m}\left(\begin{array}{cc}
w_{k+1}, & w_{k}, q \\
p, & 1,
\end{array}\right)
$$

Continuing this process, we reach

$$
D_{1}\left(\begin{array}{cc}
w_{m+k}, \\
p, & w_{m+k-1}, q \\
1, & q
\end{array}\right)=w_{m+k}
$$

Using some basic operations of determinants and Equation (30), we can easily get the following properties among the sequences $U_{n}$ and $V_{n}$.

Proposition 3. For a positive integer n, we have

$$
\begin{aligned}
U_{n+1}(p, q)-q U_{n-1}(p, q) & =V_{n}(p, q), \\
U_{n+2}(p, q)-q^{2} U_{n-2}(p, q) & =p V_{n}(p, q) \\
V_{n+1}(p, q)-q V_{n-1}(p, q) & =\left(p^{2}-4 q\right) U_{n}(p, q), \\
V_{n+2}(p, q)-q^{2} V_{n-2}(p, q) & =\left(p^{3}-4 p q\right) U_{n}(p, q) .
\end{aligned}
$$

Proof. Since we use the methods similarly, we only state how to obtain Equations (32) and (35). Applying Equation (30), we have

$$
U_{n-1}(p, q)=D_{n}\left(\begin{array}{c}
U_{0}, U_{-1, q}, q \\
p, 1, q
\end{array}\right)=D_{n}\left(\begin{array}{c}
0,-1 / q, q \\
p, 1, q
\end{array}\right),
$$


where $U_{-1}(p, q)=-q^{-1} U_{1}(p, q)=-1 / q$. Therefore,

$$
U_{n+1}(p, q)-q U_{n-1}(p, q)=\mathbb{D}_{n}(p, 1, q)-D_{n}\left(\begin{array}{c}
0,-1, q \\
p, 1, q
\end{array}\right)=D_{n}\left(\begin{array}{c}
p, 2, q \\
p, 1, q
\end{array}\right)=V_{n}(p, q) .
$$

This is Equation (32). Applying Equation (30) to $V_{n+2}$ and $V_{n-2}$, we have

$$
\begin{aligned}
& V_{n+2}(p, q)=D_{n}\left(\begin{array}{c}
V_{3}, V_{2}, q \\
p, 1, q
\end{array}\right)=D_{n}\left(\begin{array}{c}
p^{3}-3 p q, p^{2}-2 q, q \\
p, q \\
1,
\end{array}\right), \\
& V_{n-2}(p, q)=D_{n}\left(\begin{array}{c}
V_{-1}, V_{-2, q} \\
p, 1, q
\end{array}\right)=D_{n}\left(\begin{array}{c}
p / q,\left(p^{2}-2 q\right) / q^{2}, q \\
p, \\
q
\end{array}\right) .
\end{aligned}
$$

Therefore,

$$
\begin{aligned}
& V_{n+2}(p, q)-q^{2} V_{n-2}(p, q)=D_{n}\left(\begin{array}{c}
p^{3}-3 p q, p^{2}-2 q, q \\
p
\end{array}\right)-D_{n}\left(\begin{array}{c}
p q, p^{2}-2 q, q \\
p
\end{array}\right) \\
& =D_{n}\left(p_{p}^{3}-4 p q, 0, q, q\right) \\
& =\left(p^{3}-4 p q\right) D_{n}\left(\begin{array}{c}
1,0, q \\
p 1, q
\end{array}\right) \\
& =\left(p^{3}-4 p q\right) U_{n}(p, q) \text {. }
\end{aligned}
$$

This completes our proof.

These identities are generalizations of the well-known formulas between $F_{n}$ and $L_{n}$ (see [8] [Equations (5.14-16)]):

$$
\begin{aligned}
& F_{n+1}+F_{n-1}=L_{n}=F_{n+2}-F_{n-2} \\
& L_{n+1}+L_{n-1}=5 F_{n}=L_{n+2}-L_{n-2} .
\end{aligned}
$$

We derive one more identity (see [1] [Equation (3.14)]) at the end of this section.

Proposition 4. For a positive integer n, we have

$$
w_{m} U_{n+1}-q w_{m-1} U_{n}=w_{m+n} .
$$

Proof. We write the numbers $U_{n+1}$ and $U_{n}$ as their corresponding determinantal forms of order $n$.

Then,

$$
\begin{aligned}
w_{m} U_{n+1}-q w_{m-1} U_{n} & =w_{m} \mathbb{D}_{n}(p, 1, q)-q w_{m-1} D_{n}\left(\begin{array}{c}
1,0, q \\
p, 1, q
\end{array}\right) \\
& =D_{n}\left(\begin{array}{c}
p w_{m}-q w_{m-1}, w_{m}, q \\
p \\
1
\end{array}\right) \\
& =D_{n}\left(\begin{array}{c}
w_{m+1}, w_{m}, q \\
p, 1, q
\end{array}\right)
\end{aligned}
$$

Using Equation (30), the last term of the above identities becomes $w_{m+n}$.

\section{Eigenvalues of $M_{n}\left(\begin{array}{c}m, 1, q \\ p, 1, q\end{array}\right)$}

We assume that the sequence $\left(w_{n}(a, b ; p, q)\right)$ is a nontrivial second-order linear sequence, that is the parameters $a$ and $b$ are not both zeroes, and $q \neq 0$.

If $a=0$, then $b \neq 0$. Thus, we use Equation (17) to transform the determinant form of $w_{n}(a, b ; p, q)$ to the special form $D_{n}\left(\begin{array}{c}m, 1, q \\ p, 1, q\end{array}\right)$ :

$$
w_{n}(a, b ; p, q)=D_{n}\left(\begin{array}{l}
b, a, q \\
p, 1, q
\end{array}\right)=D_{n-1}\left(\begin{array}{cc}
b p-a q, b, q \\
p, & 1, q
\end{array}\right)=b D_{n-1}\left(\begin{array}{rr}
(b p-a q) / b, & 1, q \\
p, & 1, q
\end{array}\right) .
$$

If $a \neq 0$, then

$$
w_{n}(a, b ; p, q)=D_{n}\left(\begin{array}{l}
b, a, q \\
p, 1, q
\end{array}\right)=a D_{n}\left(\begin{array}{c}
b / a, 1, q \\
p, 1, q
\end{array}\right)
$$


Therefore, if we want to investigate the complex factorizations of any Horadam sequences, we only need to investigate the eigenvalues of the special matrix form $M_{n}\left(\begin{array}{c}m, 1, q, q \\ p, 1, q\end{array}\right)$.

Theorem 2. For any integer $n \geq 2$ and any complex numbers $m, p, q$ with $q \neq 0$, the eigenvalues of $M_{n}\left(\begin{array}{c}m, 1, q \\ p, 1, q\end{array}\right)$ are for $1 \leq j \leq n$,

$$
\lambda_{j}=p+2 \sqrt{q} \cos \theta_{j}
$$

with an associated eigenvector

$$
\vec{\mu}_{j}=<(p-m) q^{(k-2) / 2} \sin (k-1) \theta_{j}+q^{(k-1) / 2} \sin k \theta_{j}>_{k=1}^{n},
$$

where the parameter $\theta_{j}$ satisfies the conditions: $\theta_{j} \neq s \pi$, for any $s \in \mathbb{Z}$ and

$$
(p-m) \sin \left(n \theta_{j}\right)+\sqrt{q} \sin \left((n+1) \theta_{j}\right)=0 .
$$

Furthermore,

$$
D_{n}\left(\begin{array}{l}
m, 1, q \\
p, 1, q
\end{array}\right)=\prod_{j=1}^{n}\left(p+2 \sqrt{q} \cos \theta_{j}\right)
$$

Proof. Let $\lambda$ be an eigenvalue of the matrix $M_{n}$ with an associated eigenvector $\vec{\mu}=<\mu_{1}, \mu_{2}, \cdots, \mu_{n}>^{T}$. The equations of $M_{n} \vec{\mu}=\lambda \vec{\mu}$ are

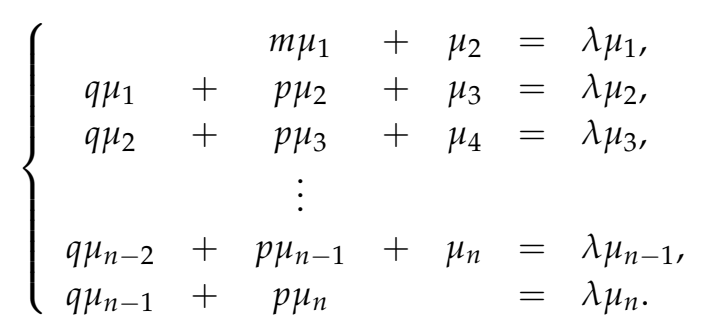

We let $\mu_{0}=0=\mu_{n+1}$. Then, the sequence $\left(\mu_{k}\right)$ satisfies

$$
q \mu_{k}+p \mu_{k+1}+\mu_{k+2}=\lambda \mu_{k+1}+f_{k+1}
$$

for $k=0,1, \ldots, n-1$, and $\mu_{0}=0=\mu_{n+1}$, where $f_{1}=(p-m) \mu_{1}, f_{k}=0$, for any nonnegative integer $k \neq 1$. Note that $\mu_{1} \neq 0$, since, if $\mu_{1}=0$, then $\vec{\mu}=\overrightarrow{\mathbf{0}}$ (the zero vector), which contradicts with the definition of an eigenvector.

Let $G(z)=\sum_{k=0}^{\infty} \mu_{k} z^{k}$ be the ordinary generating function of the sequence $\left(\mu_{k}\right)$. Multiplying $z^{k+2}$ and summing up $k$ from zero to infinity, we get

$$
\left(G(z)-\mu_{0}-\mu_{1} z\right)+(p-\lambda) z\left(G(z)-\mu_{0}\right)+q z^{2} G(z)=(p-m) \mu_{1} z^{2} .
$$

Since $\mu_{0}=0$, we get an expression of $G(z)$ from the above identity:

$$
G(z)=\frac{(p-m) \mu_{1} z^{2}+\mu_{1} z}{1+(p-\lambda) z+q z^{2}}
$$

Let

$$
r_{ \pm}=\frac{\lambda-p \pm \sqrt{(p-\lambda)^{2}-4 q}}{2 q}=\frac{\lambda-p \pm \sqrt{h}}{2 q}
$$

be the two roots of $q z^{2}+(p-\lambda) z+1=0$, where $h=(p-\lambda)^{2}-4 q$. If $h=0$, then $r_{+}=r_{-}$and the Vieta's formula gives 


$$
r_{+}+r_{-}=2 r_{+}=(\lambda-p) / q, \quad \text { and } \quad r_{+} r_{-}=r_{+}^{2}=1 / q .
$$

Therefore,

$$
q z^{2}+(p-\lambda) z+1=q\left(z-r_{+}\right)^{2}=q\left(z \mp \frac{1}{\sqrt{q}}\right)^{2}=(1 \pm \sqrt{q} z)^{2}
$$

We rewrite the right-hand side of Equation (42) as a power series expansion.

$$
\begin{aligned}
G(z) & =\left((p-m) \mu_{1} z+\mu_{1}\right) \cdot\left(\sum_{k=0}^{\infty} k( \pm \sqrt{q})^{k-1} z^{k}\right) \\
& =\sum_{k=1}^{\infty} z^{k} \mu_{1}( \pm \sqrt{q})^{k-2}[m-p+k(p-m \pm \sqrt{q})] .
\end{aligned}
$$

Comparing the coefficient of $z^{n+1}$, we have

$$
0=\mu_{n+1}=\mu_{1}( \pm \sqrt{q})^{n-1}[m-p+(n+1)(p-m \pm \sqrt{q})] .
$$

Since $\mu_{1} \neq 0 \neq q$ and $n \geq 2$, we get

$$
m-p+(p-m \pm \sqrt{q})(n+1)=0 .
$$

This formula must be valid for any $n \geq 2$, which implies that

$$
\begin{array}{ccc}
m-p=0 & \text { and } & p-m \pm \sqrt{q}=0 \\
m=p & \text { and } & q=0 .
\end{array}
$$

However, this contradicts with the fact $q \neq 0$. Therefore, $h \neq 0$. The roots $r_{+} \neq r_{-}$, and we also have the Vieta's formula:

$$
r_{+}+r_{-}=(\lambda-p) / q, \quad \text { and } \quad r_{+} r_{-}=1 / q
$$

Assume that

$$
r_{ \pm}=\epsilon \pm i \delta=\rho e^{ \pm i \theta}=\rho(\cos \theta \pm i \sin \theta)
$$

where

$$
\rho=\sqrt{\epsilon^{2}+\delta^{2}}=1 / \sqrt{q} \text { and } \cos \theta=\epsilon / \rho=(\lambda-p) /(2 \sqrt{q}) .
$$

From this assumption, we know that

$$
\lambda=p+2 \sqrt{q} \cos \theta
$$

By the method of partial fractions, we rewrite the form of $G(z)$ in Equation (42) as follows.

$$
\begin{aligned}
G(z) & =\frac{(p-m) \mu_{1} z^{2}+\mu_{1} z}{q\left(z-r_{+}\right)\left(z-r_{-}\right)} \\
& =\frac{(p-m) \mu_{1} z^{2}+\mu_{1} z}{\sqrt{h}}\left(\frac{1}{r_{-}-z}-\frac{1}{r_{+}-z}\right) \\
& =\frac{(p-m) \mu_{1} z^{2}+\mu_{1} z}{\sqrt{h}}\left(\frac{q r_{+}}{1-q r_{+} z}-\frac{q r_{-}}{1-q r_{-} z}\right) \\
& =\frac{(p-m) \mu_{1} z^{2}+\mu_{1} z}{\sqrt{h}} \sum_{k=0}^{\infty}\left[\left(q r_{+}\right)^{k+1}-\left(q r_{-}\right)^{k+1}\right] z^{k} .
\end{aligned}
$$


Using De Moivre's theorem, we get

$$
G(z)=\frac{(p-m) \mu_{1} z^{2}+\mu_{1} z}{\sqrt{h}} \sum_{k=0}^{\infty} 2 i \sin [(k+1) \theta](\rho q)^{k+1} z^{k} .
$$

Comparing the coefficients of $z^{k}$, for $1 \leq k \leq n+1$, we have

$$
\left\{\begin{aligned}
\mu_{1} & =\frac{\mu_{1} 2 i}{\sqrt{h}} \sin \theta \rho q, \\
\mu_{k} & =\frac{\mu_{1} 2 i}{\sqrt{h}}\left[(p-m) \sin [(k-1) \theta](\rho q)^{k-1}+\sin [k \theta](\rho q)^{k}\right], \quad 2 \leq k \leq n, \\
\mu_{n+1} & =\frac{\mu_{1} 2 i}{\sqrt{h}}\left[(p-m) \sin (n \theta)(\rho q)^{n}+\sin [(n+1) \theta](\rho q)^{n+1}\right] .
\end{aligned}\right.
$$

Since $\mu_{1} \neq 0$, we can rule out this factor from the first identity. Then,

$$
\sin \theta=\frac{\sqrt{h}}{\rho q 2 i} \neq 0 .
$$

This gives the restriction of $\theta$ that $\theta \neq s \pi$, for any $s \in \mathbb{Z}$. Since $\mu_{n+1}=0$, the last identity of Equation (45) gives the other restriction on $\theta$ :

$$
(p-m) \sin (n \theta)+\sqrt{q} \sin [(n+1) \theta]=0 .
$$

Furthermore, if we set $\mu_{1}=\sin \theta$, then for $2 \leq k \leq n$,

$$
\mu_{k}=(p-m) \sin [(k-1) \theta] q^{(k-2) / 2}+\sin (k \theta) q^{(k-1) / 2} .
$$

These numbers, $\mu_{k}, 1 \leq k \leq n$, form an associated eigenvector corresponding with the eigenvalue $\lambda=p+2 \sqrt{q} \cos \theta$. Since the determinant of a matrix can be found by the product of all its eigenvalues, we have

$$
D_{n}\left(\begin{array}{c}
m, 1, q \\
p, 1, q
\end{array}\right)=\prod_{j=1}^{n}\left(p+2 \sqrt{q} \cos \theta_{j}\right) .
$$

In general, the roots $\theta_{j}$ in Equation (39) are not easy to solve. To give some applications of Theorem 2, we discuss three particular cases: (1) $m=p$; (2) $m=p-\sqrt{q}$; and (3) $m=p+\sqrt{q}$. Firstly, if $m=p$, then Equation (39) becomes

$$
\sqrt{q} \sin \left[(n+1) \theta_{j}\right]=0
$$

Since $\theta_{j} \neq s \pi$, for any $s \in \mathbb{Z}$, the roots are

$$
\theta_{j}=\frac{j \pi}{n+1}, \quad \text { for } 1 \leq j \leq n
$$

Therefore, all the $n$ eigenvalues of $\mathbb{M}_{n}(p, 1, q)$ are

$$
p+2 \sqrt{q} \cos \frac{j \pi}{n+1},
$$

where $1 \leq j \leq n$. Secondly, if $m=p-\sqrt{q}$, then Equation (39) becomes

$$
\begin{aligned}
\sin \left(n \theta_{j}\right)+\sin \left[(n+1) \theta_{j}\right] & =0, \\
2 \sin \frac{(2 n+1) \theta_{j}}{2} \cos \frac{\theta_{j}}{2} & =0 .
\end{aligned}
$$


Since $\theta_{j} \neq s \pi$, for any $s \in \mathbb{Z}$, the factor $\cos \left(\theta_{j} / 2\right) \neq 0$. This implies that $\sin \left((2 n+1) \theta_{j} / 2\right)=0$. Therefore,

$$
\theta_{j}=\frac{2 j \pi}{2 n+1}, \quad \text { for } 1 \leq j \leq n .
$$

All the $n$ eigenvalues of $M_{n}\left(\begin{array}{c}p-\sqrt{q}, 1, q \\ p,\end{array}\right.$, are

$$
p+2 \sqrt{q} \cos \frac{2 j \pi}{2 n+1}
$$

where $1 \leq j \leq n$. Thirdly, if $m=p+\sqrt{q}$, then Equation (39) becomes

$$
\begin{aligned}
\sin \left[(n+1) \theta_{j}\right]-\sin \left(n \theta_{j}\right) & =0, \\
2 \sin \frac{\theta_{j}}{2} \cos \frac{(2 n+1) \theta_{j}}{2} & =0 .
\end{aligned}
$$

Since $\theta_{j} \neq s \pi$, for any $s \in \mathbb{Z}$, the factor $\sin \left(\theta_{j} / 2\right) \neq 0$. This implies that $\cos \left((2 n+1) \theta_{j} / 2\right)=0$. Therefore,

$$
\theta_{j}=\frac{(2 j-1) \pi}{2 n+1}, \quad \text { for } 1 \leq j \leq n .
$$

All the $n$ eigenvalues of $M_{n}\left(\begin{array}{c}p+\sqrt{q}, 1, q \\ p, 1, q\end{array}\right)$ are

$$
p+2 \sqrt{q} \cos \frac{(2 j-1) \pi}{2 n+1}
$$

where $1 \leq j \leq n$. We apply these three results to evaluate their corresponding determinants.

Proposition 5. Let $p, q$ be any complex numbers, with $q \neq 0$, and $n$ be any positive integer. Then, we have

$$
\begin{aligned}
\mathbb{D}_{n}(p, 1, q) & =\prod_{j=1}^{n}\left(p+2 \sqrt{q} \cos \frac{j \pi}{n+1}\right), \\
D_{n}\left(\begin{array}{c}
p-\sqrt{q}, 1, q \\
p, 1, q
\end{array}\right) & =\prod_{j=1}^{n}\left(p+2 \sqrt{q} \cos \frac{2 j \pi}{2 n+1}\right), \\
D_{n}\left(\begin{array}{c}
p+\sqrt{q}, 1, q \\
p, 1, q
\end{array}\right) & =\prod_{j=1}^{n}\left(p+2 \sqrt{q} \cos \frac{(2 j-1) \pi}{2 n+1}\right) .
\end{aligned}
$$

\section{Complex Factorizations of Some Lucas Sequences}

When we deal with the complex factorizations of $V_{n}(p, q)$, it is hard to solve $\theta_{j}$ from Equation (39) explicitly. Thus, we use another method to settle these factorizations.

The Chebyshev polynomials of the first kind $\left(T_{n}(x)\right)$ are defined by

$$
T_{0}(x)=1, \quad T_{1}(x)=x, \quad T_{n}(x)=2 x T_{n-1}(x)-T_{n-2}(x), \quad \text { for } n \geq 2 .
$$

In fact, we can write the sequence $\left(T_{n}(x)\right)$ as

$$
T_{n}(x)=w_{n}(1, x ; 2 x, 1)=D_{n}\left(\begin{array}{c}
x, 1,1 \\
2 x, 1,1
\end{array}\right) .
$$

Rivlin [9] showed that, if we set $x=\cos \theta$, then

$$
T_{n}(x)=\cos (n \theta) .
$$

Therefore, the roots of $T_{n}(x)=0$ are given by 


$$
x_{j}=\cos \theta_{j}=\cos \frac{(2 j-1) \pi}{2 n}, \quad \text { for } 1 \leq j \leq n .
$$

Please note that some methods of orthogonal polynomial sequences based on matrix calculus have recently been proposed (see $[10,11]$ and references therein).

Lemma 3. The eigenvalues of $\lambda_{j}$ of $M_{n}\left(\begin{array}{l}0,2,1 \\ 0,1,1\end{array}\right)$ are

$$
\lambda_{j}=-2 \cos \frac{(2 j-1) \pi}{2 n}, \quad \text { for } 1 \leq j \leq n .
$$

Proof. The eigenvalues of $\lambda_{j}$ of $M_{n}\left(\begin{array}{l}0,2,1 \\ 0,1,1\end{array}\right)$ are the solutions of

$$
0=\operatorname{det}\left(M_{n}\left(\begin{array}{l}
0,2,1 \\
0,1,1
\end{array}\right)-\lambda I_{n}\right)=D_{n}\left(\begin{array}{l}
-\lambda, 2,1 \\
-\lambda, 1,1
\end{array}\right)=2 D_{n}\left(\begin{array}{c}
-\lambda / 2,1,1 \\
-\lambda, 1,1
\end{array}\right) .
$$

From Equation (53), we know that $\lambda_{j}$ are solutions of

$$
T_{n}(-\lambda / 2)=0 .
$$

Using Equation (54), we have

$$
-\frac{\lambda_{j}}{2}=\cos \frac{(2 j-1) \pi}{2 n}, \quad \text { for } 1 \leq j \leq n .
$$

This gives all explicit values of $\lambda_{j}$, for $1 \leq j \leq n$.

Theorem 3. For ef $\neq 0 \neq b c$ and $2 a e f=b c d$,

$$
D_{n}\left(\begin{array}{l}
a, b, c \\
d, e, f
\end{array}\right)=\frac{b c}{2 e f} \prod_{j=1}^{n}\left(d-2 \sqrt{e f} \cos \frac{(2 j-1) \pi}{2 n}\right) .
$$

Proof. Since $e f \neq 0 \neq b c$, we apply Lemma 1 and Equation (10) to the following determinant.

$$
D_{n}\left(\begin{array}{l}
a, b, c \\
d, e, f
\end{array}\right)=D_{n}\left(\begin{array}{l}
a, 1, b c \\
d, 1, e f
\end{array}\right)=\frac{b c}{e f} D_{n}\left(\begin{array}{c}
\frac{a e f}{b b}, 1,1, e f \\
d, 1, e f
\end{array}\right)=\frac{b c}{e f} D_{n}\left(\begin{array}{l}
\frac{a e f}{b c}, \sqrt{e f}, \sqrt{e f} \\
d, \sqrt{e f}, \sqrt{e f}
\end{array}\right) .
$$

Applying $2 a e f=b c d$ into the above identity, we have

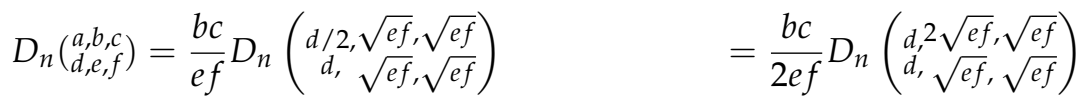

$$
\begin{aligned}
& =\frac{b c}{2 e f} \operatorname{det}\left(d I_{n}+M_{n}\left(\begin{array}{l}
0,2 \sqrt{e f}, \sqrt{e f} \\
0, \sqrt{e f}, \sqrt{e f}
\end{array}\right)\right)=\frac{b c}{2 e f} \operatorname{det}\left(d I_{n}+\sqrt{e f} M_{n}\left(\begin{array}{l}
0,2,1 \\
0,1,1
\end{array}\right)\right) .
\end{aligned}
$$

Using Lemma 3, we know that the eigenvalues of $M_{n}\left(\begin{array}{l}0,2,1 \\ 0,1,1\end{array}\right)$ are

$$
\lambda_{j}=-2 \cos \frac{(2 j-1) \pi}{2 n}, \quad \text { for } 1 \leq j \leq n .
$$

Hence, all the eigenvalues of $d I_{n}+\sqrt{e f} M_{n}\left(\begin{array}{l}0,2,1 \\ 0,1,1\end{array}\right)$ are

$$
d-2 \sqrt{e f} \cos \frac{(2 j-1) \pi}{2 n}, \quad \text { for } 1 \leq j \leq n .
$$

This completes our proof. 
For any sequence $\left(w_{n}(a, b ; p, q)\right)$, a subsequence $\left(S_{n}\right)=\left(w_{k n+t}\right)$ with indices in an arithmetic sequence is again a second-order recurrence sequence (see [6] [Lemma 8]). Moreover,

$$
\left(w_{k n+t}\right)=\left(S_{n}\right)=w_{n}\left(w_{t}, w_{k+t} ; v_{k}, q^{k}\right) .
$$

Therefore, we have

$$
U_{k n+k}=w_{n}\left(U_{k}, U_{2 k} ; V_{k}, q^{k}\right)=D_{n}\left(\begin{array}{c}
U_{2 k}, U_{k}, q^{k} \\
V_{k}, 1, q^{k}
\end{array}\right) .
$$

We use the fact (see [1] [Equation (4.10)]) that

$$
U_{2 n}=U_{n} V_{n}
$$

and Equation (10) in the above identity.

$$
U_{k n+k}=U_{k} \cdot \mathbb{D}_{n}\left(V_{k}, 1, q^{k}\right) .
$$

Moreover, we can apply Equation (49) and $\cos (\pi-x)=-\cos (x)$ to give a complex factorization of $U_{k n+k}$.

Proposition 6. For any integers $n, k$ with $n \geq 1$, the subsequence $\left(U_{k n+k}\right)_{n=1}^{\infty}$ has the following determinantal representation and a complex factorization:

$$
\begin{aligned}
U_{k n+k}=U_{k} \cdot \mathbb{D}_{n}\left(V_{k}, 1, q^{k}\right) & =U_{k} \prod_{j=1}^{n}\left(V_{k}+2 \sqrt{q^{k}} \cos \frac{j \pi}{n+1}\right) \\
& =U_{k} \prod_{j=1}^{n}\left(V_{k}-2 \sqrt{q^{k}} \cos \frac{j \pi}{n+1}\right) .
\end{aligned}
$$

Using Equation (57), the subsequence $\left(U_{2 k n+k}\right)$ can be handled by the following process.

$$
U_{2 k n+k}=w_{n}\left(U_{k}, U_{3 k} ; V_{2 k}, q^{2 k}\right)=D_{n}\left(\begin{array}{c}
U_{3 k}, U_{k}, q^{2 k} \\
V_{2 k}, 1, q^{2 k}
\end{array}\right) .
$$

We use the fact that [1] [Equation (4.13)]

$$
U_{3 n}=U_{n}\left(V_{2 n}+q^{n}\right)
$$

and Equation (10) in the above identity.

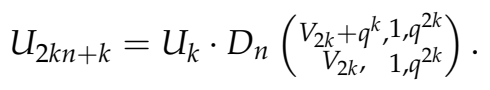

Moreover, we can apply Equations (50) and (51) to give a complex factorization of $U_{2 k n+k}$.

Proposition 7. For any integers $n, k$ with $n \geq 1$, the subsequence $\left(U_{2 k n+k}\right)_{n=1}^{\infty}$ has the following determinantal representation and a complex factorization:

$$
\begin{aligned}
U_{2 k n+k} & =U_{k} \cdot D_{n}\left(\begin{array}{c}
V_{2 k}+q^{k}, 1, q^{2 k} \\
V_{2 k}, 1, q^{2 k}
\end{array}\right) \\
& = \begin{cases}U_{k} \prod_{j=1}^{n}\left(V_{2 k}-2 q^{k} \cos \frac{2 j \pi}{2 n+1}\right), & \text { if } q<0, k \text { is odd }, \\
U_{k} \prod_{j=1}^{n}\left(V_{2 k}+2 q^{k} \cos \frac{(2 j-1) \pi}{2 n+1}\right), & \text { otherwise. }\end{cases}
\end{aligned}
$$


Now, we deal the subsequences of $\left(V_{n}\right)$. Firstly, we consider the subsequence $\left(V_{k n}\right)$. From Equation (57), we have

$$
V_{k n}=w_{n}\left(2, V_{k} ; V_{k}, q^{k}\right)=D_{n}\left(\begin{array}{c}
V_{k}, 2, q^{k} \\
V_{k}, 1, q^{k}
\end{array}\right) .
$$

We apply Theorem 3 to the above identity and we can give a complex factorization of $V_{k n}$ as follows.

Proposition 8. For any integers $n, k$ with $n \geq 1$, the subsequence $\left(V_{k n}\right)_{n=1}^{\infty}$ has the following determinantal representation and a complex factorization:

$$
V_{k n}=D_{n}\left(\begin{array}{c}
V_{k}, 2, q^{k} \\
V_{k}, 1, q^{k}
\end{array}\right)=\prod_{j=1}^{n}\left(V_{k}-2 \sqrt{q^{k}} \cos \frac{(2 j-1) \pi}{2 n}\right) .
$$

Using Equation (57), the subsequence $\left(V_{2 k n+k}\right)$ can be handled by the following process.

$$
V_{2 k n+k}=w_{n}\left(V_{k}, V_{3 k} ; V_{2 k}, q^{2 k}\right)=D_{n}\left(\begin{array}{c}
V_{3 k}, V_{k}, q^{2 k} \\
V_{2 k}, 1, q^{2 k}
\end{array}\right) .
$$

We use the fact the ([1], Equation (4.14))

$$
V_{3 n}=V_{n}\left(V_{2 n}-q^{n}\right)
$$

and Equation (10) in the above identity.

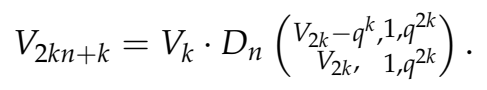

Moreover, we can apply Equations (50) and (51) to give a complex factorization of $V_{2 k n+k}$.

Proposition 9. For any integers $n, k$ with $n \geq 1$, the subsequence $\left(V_{2 k n+k}\right)_{n=1}^{\infty}$ has the following determinantal representation and a complex factorization:

$$
\begin{aligned}
V_{2 k n+k} & =V_{k} \cdot D_{n}\left(\begin{array}{cl}
V_{2 k}-q^{k}, 1, q^{2 k} \\
V_{2 k}, 1, q^{2 k}
\end{array}\right) \\
& = \begin{cases}V_{k} \prod_{j=1}^{n}\left(V_{2 k}-2 q^{k} \cos \frac{(2 j-1) \pi}{2 n+1}\right), & \text { if } q<0, k \text { is odd }, \\
V_{k} \prod_{j=1}^{n}\left(V_{2 k}+2 q^{k} \cos \frac{2 j \pi}{2 n+1}\right), & \text { otherwise. }\end{cases}
\end{aligned}
$$

\section{Some Applications and Concluding Remarks}

In this section, we give some applications on some famous sequences: Fibonacci numbers $F_{n}$, Lucas numbers $L_{n}$, Pell numbers $P_{n}$, and Pell-Lucas numbers $Q_{n}$.

Firstly, we give the applications of Proposition 6. Let $p=-q=1, k=2 m$ in Equation (58) where $m \in \mathbb{Z}$, we have (see [5] [Equation (3)])

$$
F_{2 m(n+1)}=F_{2 m} \cdot \mathbb{D}_{n}\left(L_{2 m}, 1,1\right)=F_{2 m} \prod_{j=1}^{n}\left(L_{2 m}+2 \cos \frac{j \pi}{n+1}\right)
$$

We find that $F_{2 n+2}=\mathbb{D}_{n}(3,1,1)$ in [3] and we list the complex factorization with $m=1$ in the above identity (note that $F_{2}=1, L_{2}=3$ ):

$$
F_{2 n+2}=\mathbb{D}_{n}(3,1,1)=\prod_{j=1}^{n}\left(3+2 \cos \frac{j \pi}{n+1}\right) .
$$


Let $p=-q=1, k=2 m+1$ in Equation (58), where $m \in \mathbb{Z}$; we have

$$
F_{(2 m+1)(n+1)}=F_{2 m+1} \cdot \mathbb{D}_{n}\left(L_{2 m+1}, 1,-1\right)=F_{2 m+1} \prod_{j=1}^{n}\left(L_{2 m+1}+2 i \cdot \cos \frac{j \pi}{n+1}\right) .
$$

We find that $F_{n+1}=\mathbb{D}_{n}(1, i, i)=\mathbb{D}_{n}(1,1,-1)$ in $[2,4]$. We list two identities with $m=0$ and $m=1$ :

$$
\begin{gathered}
F_{n+1}=\mathbb{D}_{n}(1,1,-1)=\prod_{j=1}^{n}\left(1+2 i \cdot \cos \frac{j \pi}{n+1}\right), \\
F_{3 n+3}=2 \cdot \mathbb{D}_{n}(4,1,-1)=2 \prod_{j=1}^{n}\left(4+2 i \cdot \cos \frac{j \pi}{n+1}\right) .
\end{gathered}
$$

If we set $p=2, q=-1$, then $U_{n}(2,-1)=P_{n}$ and $V_{n}(2,-1)=Q_{n}$. Substituting these parameters into Equation (58), we have

$$
\begin{gathered}
P_{2 m(n+1)}=P_{2 m} \cdot \mathbb{D}_{n}\left(Q_{2 m}, 1,1\right) \quad=P_{2 m} \prod_{j=1}^{n}\left(Q_{2 m}+2 \cos \frac{j \pi}{n+1}\right), \\
P_{(2 m+1)(n+1)}=P_{2 m+1} \cdot \mathbb{D}_{n}\left(Q_{2 m+1}, 1,-1\right)=P_{2 m+1} \prod_{j=1}^{n}\left(Q_{2 m+1}+2 i \cdot \cos \frac{j \pi}{n+1}\right) .
\end{gathered}
$$

Here, we list the representations for $P_{n+1}$ and $P_{2 n+2}$ :

$$
\begin{gathered}
P_{n+1}=\mathbb{D}_{n}(2,1,-1)=\prod_{j=1}^{n}\left(2+2 i \cdot \cos \frac{j \pi}{n+1}\right), \\
P_{2 n+2}=2 \mathbb{D}_{n}(6,1,1)=2 \prod_{j=1}^{n}\left(6+2 \cos \frac{j \pi}{n+1}\right) .
\end{gathered}
$$

Secondly, we give the applications of Proposition 7. Let $p=-q=1$ and $k$ be an even or odd integer in Equation (60); we have the identities among Fibonacci numbers:

$$
\begin{gathered}
F_{2 m(2 n+1)}=F_{2 m} \cdot D_{n}\left(\begin{array}{c}
L_{4 m}+1,1,1 \\
L_{4 m}, 1,1
\end{array}\right)=F_{2 m} \prod_{j=1}^{n}\left(L_{4 m}+2 \cos \frac{(2 j-1) \pi}{2 n+1}\right), \\
F_{(2 m+1)(2 n+1)}=F_{2 m+1} \cdot D_{n}\left(\begin{array}{c}
L_{4 m+2}-1,1,1 \\
L_{4 m+2}, 1,1
\end{array}\right)=F_{2 m+1} \prod_{j=1}^{n}\left(L_{4 m+2}+2 \cos \frac{2 j \pi}{2 n+1}\right) .
\end{gathered}
$$

We find that $F_{2 n+1}=D_{n}\left(\begin{array}{l}2,1,1 \\ 3,1,1\end{array}\right)$ appeared in [3] and we list the representations for $F_{2 n+1}$ and $F_{4 n+2}$ :

$$
\begin{aligned}
& F_{2 n+1}=D_{n}\left(\begin{array}{l}
2,1,1 \\
3,1,1
\end{array}\right)=\prod_{j=1}^{n}\left(3+2 \cos \frac{2 j \pi}{2 n+1}\right), \\
& F_{4 n+2}=D_{n}\left(\begin{array}{l}
8,1,1 \\
7,1,1
\end{array}\right)=\prod_{j=1}^{n}\left(7+2 \cos \frac{(2 j-1) \pi}{2 n+1}\right) .
\end{aligned}
$$

If we change the values of the parameters $p$ and $q$ to $p=2, q=-1$. Then, Equation (60) becomes

$$
\begin{aligned}
& P_{2 m(2 n+1)}=P_{2 m} \cdot D_{n}\left(\begin{array}{c}
Q_{4 m}+1,1,1 \\
Q_{4 m}, 1,1
\end{array}\right)=P_{2 m} \prod_{j=1}^{n}\left(Q_{4 m}+2 \cos \frac{(2 j-1) \pi}{2 n+1}\right), \\
& P_{(2 m+1)(2 n+1)}=P_{2 m+1} \cdot D_{n}\left(\begin{array}{c}
Q_{4 m+2}-1,1,1 \\
Q_{4 m+2}, 1,1
\end{array}\right)=P_{2 m+1} \prod_{j=1}^{n}\left(Q_{4 m+2}+2 \cos \frac{2 j \pi}{2 n+1}\right) \text {. }
\end{aligned}
$$


We list the representations of $P_{2 n+1}$ and $P_{4 n+2}$ :

$$
\begin{aligned}
& P_{2 n+1}=D_{n}\left(\begin{array}{c}
5,1,1 \\
6,1,1
\end{array}\right)=\prod_{j=1}^{n}\left(6+2 \cos \frac{2 j \pi}{2 n+1}\right) \\
& P_{4 n+2}=2 \cdot D_{n}\left(\begin{array}{l}
35,1,1 \\
34,1,1
\end{array}\right)=2 \prod_{j=1}^{n}\left(34+2 \cos \frac{(2 j-1) \pi}{2 n+1}\right) .
\end{aligned}
$$

Thirdly, we give the applications of Proposition 8. Let $p=-q=1$ in Equation (61); we have the identities among Lucas numbers:

$$
\begin{gathered}
L_{2 m n}=D_{n}\left(\begin{array}{l}
L_{2 m}, 2,1 \\
L_{2 m}, 1,1
\end{array}\right)=\prod_{j=1}^{n}\left(L_{2 m}-2 \cdot \cos \frac{(2 j-1) \pi}{2 n}\right), \\
L_{(2 m+1) n}=D_{n}\left(\begin{array}{l}
L_{2 m+1,2,-1} \\
L_{2 m+1,1},-1
\end{array}\right)=\prod_{j=1}^{n}\left(L_{2 m+1}-2 i \cdot \cos \frac{(2 j-1) \pi}{2 n}\right) .
\end{gathered}
$$

We find that $L_{n}=2 \cdot D_{n}\left(\begin{array}{c}1 / 2, i, i \\ 1, i, i\end{array}\right)$ in [7] [Equation (3.3)] and also its complex factorization in [7] [Equation (1.2)]. Note that, by Lemma 1 and Equation (10), we know that the determinant is equivalent to our determinant form $D_{n}\left(\begin{array}{l}1,2,-1 \\ 1,1,-1\end{array}\right)$. The following we list the representations of $L_{n}$ and $L_{2 n}$ :

$$
\begin{gathered}
L_{n}=D_{n}\left(\begin{array}{c}
1,2,-1 \\
1,1,-1
\end{array}\right)=\prod_{j=1}^{n}\left(1-2 i \cdot \cos \frac{(2 j-1) \pi}{2 n}\right) \\
L_{2 n}=D_{n}\left(\begin{array}{l}
3,2,1 \\
3,1,1
\end{array}\right)=\prod_{j=1}^{n}\left(3-2 \cos \frac{(2 j-1) \pi}{2 n}\right)
\end{gathered}
$$

Now, we consider the Pell-Lucas numbers $Q_{n}$, that is, we set $p=2, q=-1$ in Equation (61).

$$
\begin{gathered}
Q_{2 m n}=D_{n}\left(\begin{array}{l}
Q_{2 m}, 2,1 \\
Q_{2 m}, 1,1
\end{array}\right)=\prod_{j=1}^{n}\left(Q_{2 m}-2 \cdot \cos \frac{(2 j-1) \pi}{2 n}\right), \\
Q_{(2 m+1) n}=D_{n}\left(\begin{array}{l}
Q_{2 m+1}, 2,-1 \\
Q_{2 m+1}, 1,-1
\end{array}\right)=\prod_{j=1}^{n}\left(Q_{2 m+1}-2 i \cdot \cos \frac{(2 j-1) \pi}{2 n}\right) .
\end{gathered}
$$

We list the representations of $Q_{n}$ and $Q_{2 n}$.

$$
\begin{gathered}
Q_{n}=D_{n}\left(\begin{array}{c}
2,2,-1 \\
2,1,-1
\end{array}\right)=\prod_{j=1}^{n}\left(2-2 i \cdot \cos \frac{(2 j-1) \pi}{2 n}\right), \\
Q_{2 n}=D_{n}\left(\begin{array}{c}
6,2,1 \\
6,1,1
\end{array}\right)=\prod_{j=1}^{n}\left(6-2 \cos \frac{(2 j-1) \pi}{2 n+1}\right) .
\end{gathered}
$$

At last, we give the applications of Proposition 9. Let $p=-q=1$ in Equation (62), we have the identities among Lucas numbers:

$$
\begin{gathered}
L_{2 m(2 n+1)}=L_{2 m} \cdot D_{n}\left(\begin{array}{r}
L_{4 m}-1,1,1 \\
L_{4 m}, 1,1
\end{array}\right)=L_{2 m} \prod_{j=1}^{n}\left(L_{4 m}+2 \cos \frac{2 j \pi}{2 n+1}\right), \\
L_{(2 m+1)(2 n+1)}=L_{2 m+1} \cdot D_{n}\left(\begin{array}{r}
L_{4 m+2}+1,1,1 \\
L_{4 m+2}, 1,1
\end{array}\right)=L_{2 m+1} \prod_{j=1}^{n}\left(L_{4 m+2}+2 \cos \frac{(2 j-1) \pi}{2 n+1}\right) .
\end{gathered}
$$

We list the representations of $L_{2 n+1}$ and $L_{4 n+2}$. 


$$
\begin{aligned}
& L_{2 n+1}=D_{n}\left(\begin{array}{l}
4,1,1,1 \\
3,1,1
\end{array}\right)=\prod_{j=1}^{n}\left(3+2 \cos \frac{(2 j-1) \pi}{2 n+1}\right), \\
& L_{4 n+2}=3 \cdot D_{n}(6,1,1,1)=3 \prod_{j=1}^{n}\left(7+2 \cos \frac{2 j \pi}{2 n+1}\right) .
\end{aligned}
$$

Setting $p=2, q=-1$ in Equation (62), we have

$$
\begin{aligned}
& Q_{2 m(2 n+1)}=Q_{2 m} \cdot D_{n}\left(\underset{Q_{4 m}, 1,1}{Q_{4 m}-1,1,1}\right)=Q_{2 m} \prod_{j=1}^{n}\left(Q_{4 m}+2 \cos \frac{2 j \pi}{2 n+1}\right), \\
& Q_{(2 m+1)(2 n+1)}=Q_{2 m+1} \cdot D_{n}\left(\begin{array}{c}
Q_{4 m+2}+1,1,1 \\
Q_{4 m+2}, 1,1
\end{array}\right)=Q_{2 m+1} \prod_{j=1}^{n}\left(Q_{4 m+2}+2 \cos \frac{(2 j-1) \pi}{2 n+1}\right) .
\end{aligned}
$$

The representations of $Q_{2 n+1}$ and $Q_{4 n+2}$ are

$$
\begin{aligned}
& Q_{2 n+1}=2 \cdot D_{n}\left(\begin{array}{c}
7,1,1 \\
6,1,1
\end{array}\right)=2 \prod_{j=1}^{n}\left(6+2 \cos \frac{(2 j-1) \pi}{2 n+1}\right), \\
& Q_{4 n+2}=6 \cdot D_{n}\left(\begin{array}{l}
33,1,1,1 \\
34,1,1
\end{array}\right)=6 \prod_{j=1}^{n}\left(34+2 \cos \frac{2 j \pi}{2 n+1}\right) .
\end{aligned}
$$

Cahill and Narayan [5] constructed two families of tridiagonal matrices whose determinants are $F_{k n+t}$ and $L_{k n+t}$. The $n \times n$ symmetric tridiagonal matrices $M$ whose elements are given by $m_{1,1}=F_{k+t}, m_{2,2}=\left\lceil F_{2 k+t} / F_{k+t}\right\rceil, m_{1,2}=m_{2,1}=\sqrt{m_{2,2} F_{k+t}-F_{2 k+t}}, m_{j, j}=L_{k}$, where $3 \leq j \leq n$, $m_{j, j+1}=m_{j+1, j}=\sqrt{(-1)^{k}}$, where $2 \leq j<k$. That is

$$
M=\left[\begin{array}{cccccc}
F_{k+t} & \sqrt{m_{2,2} F_{k+t}-F_{2 k+t}} & 0 & 0 & \cdots & 0 \\
\sqrt{m_{2,2} F_{k+t}-F_{2 k+t}} & \left\lceil F_{2 k+t} / F_{k+t}\right\rceil & \sqrt{(-1)^{k}} & 0 & \cdots & 0 \\
0 & \sqrt{(-1)^{k}} & L_{k} & \sqrt{(-1)^{k}} & \cdots & 0 \\
0 & 0 & \sqrt{(-1)^{k}} & L_{k} & \cdots & 0 \\
\vdots & \vdots & \vdots & \vdots & \ddots & \vdots \\
0 & 0 & 0 & 0 & \cdots & L_{k}
\end{array}\right]
$$

Maybe we can say that they use three layers at the top-left of the matrix. There are two layers at the top-left in our matrix $M_{n}\left(\begin{array}{l}a, b, c \\ d, e, f\end{array}\right)$. Using a similar spirit as our notations, we may write their results as follows. (see [5] [Theorem 2-3])

$$
\begin{gathered}
F_{k n+t}=D_{n}\left(\begin{array}{ccc}
F_{k+t,}, & \sqrt{m_{2,2} F_{k+t}-F_{2 k+t}}, & \sqrt{m_{2,2} F_{k+t}-F_{2 k+t}} \\
\left\lceil F_{2 k+t} / F_{k+t}\right\rceil, & \sqrt{(-1)^{k}}, & \sqrt{(-1)^{k}} \\
L_{k}, & \sqrt{(-1)^{k}}, & \sqrt{(-1)^{k}}
\end{array}\right), \\
L_{k n+t}=D_{n}\left(\begin{array}{ccc}
L_{k+t,} & \sqrt{t_{2,2} L_{k+t}-L_{2 k+t}}, & \sqrt{t_{2,2} L_{k+t}-L_{2 k+t}} \\
\left\lceil L_{2 k+t} / L_{k+t}\right\rceil, & \sqrt{(-1)^{k}}, & \sqrt{(-1)^{k}} \\
L_{k}, & \sqrt{(-1)^{k}}, & \sqrt{(-1)^{k}}
\end{array}\right),
\end{gathered}
$$

where $t_{2,2}=\left\lceil L_{2 k+t} / L_{k+t}\right\rceil$. In this paper, we get simpler forms of the values $F_{k n+t}$ and $L_{k n+t}$.

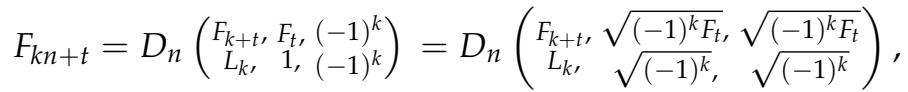

$$
\begin{aligned}
& L_{k n+t}=D_{n}\left(\begin{array}{ccc}
L_{k+t}, & L_{t}, & (-1)^{k} \\
L_{k}, & 1, & (-1)^{k}
\end{array}\right)=D_{n}\left(\begin{array}{ccc}
L_{k+t}, & \sqrt{(-1)^{k} L_{t}}, & \sqrt{(-1)^{k} L_{t}} \\
L_{k}, & \sqrt{(-1)^{k}}, & \sqrt{(-1)^{k}}
\end{array}\right) .
\end{aligned}
$$


Note that, if we allow $k$ and $t$ being all integers, our evaluations also cover all results in [12]. However, we think that, if we use three layers forms $D_{n}\left(\begin{array}{l}a, b, c \\ d, e, f \\ g, h, k\end{array}\right)$, then we might be able to deal with more complicated cases. However, this is another story.

Funding: This research was funded by the Ministry of Science and Technology, Taiwan, R.O.C. grant number MOST 108-2115-M-845-001.

Conflicts of Interest: The author declares no conflict of interest.

\section{References}

1. Horadam, A.F. Basic properties of a certain generalized sequence of numbers. Fibonacci Quart. 1965, 3, 161-176.

2. Strang, G.; Borre, K. Linear Algebra, Geodesy and GPS; Wellesley-Cambridge: Wellesley, MA, USA, 1997.

3. Strang, G. Introduction to Linear Algebra, 5th ed.; Wellesley-Cambridge: Wellesley, MA, USA, 2016.

4. Cahill, N.D.; D’Errico, J.R.; Narayan, D.A.; Narayan, J.Y. Fibonacci determinants. Coll. Math. J. 2002, 33, 221-225. [CrossRef]

5. Cahill, N.D.; Narayan, D.A. Fibonacci and Lucas numbers as tridiagonal matrix determinants. Fibonacci Quart. 2004, 42, 216-221.

6. Chen, K.-W.; Pan, Y.-R. Greatest common divisors of shifted Horadam sequences. J. Integer Seq. 2020, $23,3$.

7. Cahill, N.D.; D'Errico, J.R.; Spence, J.P. Complex factorizations of the Fibonacci and Lucas numbers. Fibonacci Quart. 2003, 41, 13-19.

8. Koshy, T. Fibonacci and Lucas Numbers with Applications; John Willey \& Sons, Inc.: Hoboken, NJ, USA, 2001.

9. Rivlin, T.J. The Chebyshev Polynomials: From Approximation Theory to Algebra and Number Theory; John Wiley \& Sons: New York, NY, USA, 1974.

10. Dominici, D. Matrix factorizations and orthogonal polynomials. Random Matrices Theory Appl. 2020, 9, 1-33. [CrossRef]

11. Costabile, F.A.; Gualtieri, M.I.; Napoli, A. Matrix calculus-based approach to orthogonal polynomial sequences. Mediterr. J. Math. 2020, 17, 118. [CrossRef]

12. Nalli, A.; Civciv, H. A generalization of tridiagonal matrix determinants, Fibonacci and Lucas numbers. Chaos Solitons Fractals 2009, 40, 355-361. [CrossRef]

Publisher's Note: MDPI stays neutral with regard to jurisdictional claims in published maps and institutional affiliations.

(C) 2020 by the author. Licensee MDPI, Basel, Switzerland. This article is an open access article distributed under the terms and conditions of the Creative Commons Attribution (CC BY) license (http:// creativecommons.org/licenses/by/4.0/). 